\title{
A New Similarity Measure between Intuitionistic Fuzzy Sets and Its Application to Pattern Recognition
}

\author{
Yafei Song, Xiaodan Wang, Lei Lei, and Aijun Xue \\ School of Air and Missile Defense, Air Force Engineering University, Xian 710051, China \\ Correspondence should be addressed to Yafei Song; yafei_song@163.com
}

Received 22 April 2014; Accepted 13 August 2014; Published 27 August 2014

Academic Editor: Maria A. Ragusa

Copyright ( 2014 Yafei Song et al. This is an open access article distributed under the Creative Commons Attribution License, which permits unrestricted use, distribution, and reproduction in any medium, provided the original work is properly cited.

\begin{abstract}
As a generation of ordinary fuzzy set, the concept of intuitionistic fuzzy set (IFS), characterized both by a membership degree and by a nonmembership degree, is a more flexible way to cope with the uncertainty. Similarity measures of intuitionistic fuzzy sets are used to indicate the similarity degree between intuitionistic fuzzy sets. Although many similarity measures for intuitionistic fuzzy sets have been proposed in previous studies, some of those cannot satisfy the axioms of similarity or provide counterintuitive cases. In this paper, a new similarity measure and weighted similarity measure between IFSs are proposed. It proves that the proposed similarity measures satisfy the properties of the axiomatic definition for similarity measures. Comparison between the previous similarity measures and the proposed similarity measure indicates that the proposed similarity measure does not provide any counterintuitive cases. Moreover, it is demonstrated that the proposed similarity measure is capable of discriminating difference between patterns.
\end{abstract}

\section{Introduction}

Since it was proposed by Zadeh [1], the theory of fuzzy set (FS) has achieved great success due to its capability of handling uncertainty. Therefore, over the last decades, several higher order fuzzy sets have been introduced in the literature. Intuitionistic fuzzy set (IFS), as one of the higher order fuzzy sets, was proposed by Atanassov [2] to deal with vagueness. The main advantage of the IFS is its property to cope with the uncertainty that may exist due to information impression. Because it assigns to each element a membership degree, a nonmembership degree, and a hesitation degree, IFS constitutes an extension of Zadeh's fuzzy set which only assigns to each element a membership degree [3]. So IFS is regarded as a more effective way to deal with vagueness than fuzzy set. Although Gau and Buehrer later presented vague set [4], it was pointed out by Bustince and Burillo that the notion of vague sets was the same as that of IFS [5].

The definition of similarity measure between two IFSs is one of the most interesting topics in IFSs theory. A similarity measure is defined to compare the information carried by IFSs. Measures of similarity between IFSs, as an important tool for decision making, pattern recognition, machine learning, and image processing, have received much attention in recent years $[6,7]$. Many similarity measures have been proposed. A few of them come from the wellknown distance measures. The first study was carried out by Szmidt and Kacprzyk [8] extending the well-known distances measures, such as the Hamming distance and the Euclidian distance, to IFS environment and comparing them with the approaches used for ordinary fuzzy sets. However, Wang and Xin [9] implied that the distance measures of Szmidt and Kacprzyk [8] were not effective in some cases. Therefore, several new distance measures were proposed and applied to pattern recognition. Grzegorzewski [10] also extended the Hamming distance, the Euclidean distance, and their normalized counterparts to IFS environment. Later, Chen [11] pointed out that some errors existed in Grzegorzewski [10] by showing some counterexamples. Hung and Yang [12] extended the Hausdorff distance to IFSs and proposed three similarity measures.

On the other hand, instead of extending the well-known measures, some studies defined new similarity measures for IFSs. Dengfeng and Chuntian [13] suggested a new similarity 
measure for IFSs based on the membership degree and the nonmembership degree. Mitchell [14] showed that the similarity measure of Dengfeng and Chuntian [13] had some counterintuitive cases and modified the similarity measure based on statistical point of view. Moreover, Liang and Shi [15] presented some examples to show that the similarity measure of Dengfeng and Chuntian [13] was not reasonable for some conditions and therefore proposed several new similarity measures for IFSs. Li et al. [16] analyzed, compared, and summarized the existing similarity measures between IFSs/vague sets by their counterintuitive examples in pattern recognition. Ye [7] conducted a similar comparative study of the existing similarity measures between IFSs and proposed a cosine similarity measure and a weighted cosine similarity measure. Hwang et al. [17] proposed a similarity measure for IFSs in which Sugeno integral was used for aggregation. The proposed similarity measure was applied to clustering problem. $\mathrm{Xu}[18]$ introduced a series of similarity measures for IFSs and applied them to multiple attribute decision making problem based on intuitionistic fuzzy information. Xu and Chen [19] introduced a series of distance and similarity measures, which are various combinations and generalizations of the weighted Hamming distance, the weighted Euclidean distance, and the weighted Hausdorff distance. Xu and Yager [20] developed a similarity measure between IFSs and applied the developed similarity measure for consensus analysis in group decision making based on intuitionistic fuzzy preference relations. Xia and $\mathrm{Xu}[6]$ proposed a series of distance measures based on the intuitionistic fuzzy point operators. In addition to these studies, some works have been interested in relationships between distance measure, similarity measure, and entropy of IFSs. Zeng and Guo [21] investigated the relationship among the normalized distance, the similarity measure, the inclusion measure, and the entropy of interval-valued fuzzy sets. It was also showed that the similarity measure, the inclusion measure, and the entropy of interval-valued fuzzy sets could be induced by the normalized distance of interval-valued fuzzy sets based on their axiomatic definitions. Wei et al. [22] introduced an entropy measure generalizing the existing entropy measures for IFS and IFSs. Also an approach was introduced to construct similarity measures using entropy measures for IFS and IFSs. Boran and Akay [23] proposed a new general type of similarity measure for IFS with two parameters, expressing $L_{p}$ norm and the level of uncertainty, respectively. This similarity measure can also make sense in terms of counterintuitive cases.

Moreover, Zhang and $\mathrm{Yu}$ [24] presented a new distance (or similarity) measure based on interval comparison, where the IFSs were, respectively, transformed into the symmetric triangular fuzzy numbers. Comparison with the widely used methods indicated that the proposed method contained more information, with much less loss of information. Li et al. [25] introduced an axiomatic definition of the similarity measure of IFSs. The relationship between the entropy and the similarity measure of IFS was investigated in detail. It was proved that the similarity measure and the entropy of IFS can be transformed into each other based on their axiomatic definitions. Farhadinia [26] generalized results on the entropy of interval-valued fuzzy sets (IVFSs) based on the intuitionistic distance and its relationship with similarity measure. Based on the set of new axioms, he also proved some theorems that entropy and similarity measure for IVFSs can be transformed by each other in a general way. Li [27] defined two dissimilarity measures between intuitionistic fuzzy sets of a finite set, and it was proved that both of the measures are metrical. Papakostas et al. [28] investigated the main theoretical and computational properties of the measures as well as the relationships between them. A comparison of the distance and similarity measures was carried out by them, from a pattern recognition point of view.

Among similarity measures proposed by other authors, some of those, however, cannot satisfy the axioms of similarity or provide counterintuitive cases or are produced by complex formats. Therefore, we propose new similarity measures with relative simple expression. The proposed similarity measure depends on the triplet, membership degree, nonmembership degree, and hesitation margin. This paper proves that the proposed measures satisfy the properties of the axiomatic definition for similarity measures. In addition, several numerical examples are provided to compare the proposed measure with a number of existing measures.

The remainder of this paper is organized as follows. Section 2 recalls the definitions related to the IFSs and lists the properties that a distance measure for IFSs and a similarity measure for IFSs should possess. In Section 3, the existing similarity measures for IFSs will be recalled. The new similarity measure, together with its proofs, is presented in Section 4. Comparison between the proposed similarity measure and the existing similarity measures is carried out in Section 5. The application of the proposed similarity measure to pattern recognition is presented in Section 6, followed by the conclusion of this paper in Section 7.

\section{Preliminaries}

In this section, we briefly recall the basic concepts related to IFS and then list the properties of the axiomatic definition for similarity measures.

Definition 1. Let $X=\left\{x_{1}, x_{2}, \ldots, x_{n}\right\}$ be a universe of discourse; then, a fuzzy set $A$ in $X$ is defined as follows [1]:

$$
A=\left\{\left\langle x, \mu_{A}(x)\right\rangle \mid x \in X\right\},
$$

where $\mu_{A}(x): X \rightarrow[0,1]$ is the membership degree.

Definition 2. An IFS $A$ in $X$ defined by Atanassov can be written as [2]

$$
A=\left\{\left\langle x, \mu_{A}(x), v_{A}(x)\right\rangle \mid x \in X\right\},
$$

where $\mu_{A}(x): X \rightarrow[0,1]$ and $v_{A}(x): X \rightarrow[0,1]$ are membership degree and nonmembership degree, respectively, with the condition

$$
0 \leq \mu_{A}(x)+v_{A}(x) \leq 1 .
$$

$\pi_{A}(x)$ determined by the following expression:

$$
\pi_{A}(x)=1-\mu_{A}(x)-v_{A}(x),
$$


is called the hesitancy degree of the element $x \in X$ to the set $A$, and $\pi_{A}(x) \in[0,1]$, for all $x \in X$.

$\pi_{A}(x)$ is also called the intuitionistic index of $x$ to $A$. Greater $\pi_{A}(x)$ indicates more vagueness on $x$. Obviously, when $\pi_{A}(x)=0$, for all $x \in X$, the IFS degenerates into an ordinary fuzzy set.

In the sequel, the couple $\left\langle\mu_{A}(x), v_{A}(x)\right\rangle$ is called an IFS or intuitionistic fuzzy value (IFV) for clarity. Let IFSs $(X)$ denote the set of all IFSs in $X$.

Definition 3. For $A \in \operatorname{IFSs}(X)$ and $B \in \operatorname{IFSs}(X)$, some relations between them are defined as

(R1) $A \subset B$ if and only if for all $x \in X \mu_{A}(x) \leq$ $\mu_{B}(x), v_{A}(x) \geq v_{B}(x)$;

(R2) $A=B$ if and only if for all $x \in X \mu_{A}(x)=$ $\mu_{B}(x), v_{A}(x)=v_{B}(x)$

(R3) $A^{C}=\left\{\left\langle x, v_{A}(x), \mu_{A}(x)\right\rangle \mid x \in X\right\}$, where $A^{C}$ is the complement of $A$.

Definition 4. Let $D$ denote the mapping $D:$ IFS $\times$ IFS $\rightarrow$ $[0,1]$; if $D(A, B)$ satisfies the following properties, $D(A, B)$ is called a distance between $A \in \operatorname{IFSs}(X)$ and $B \in \operatorname{IFSs}(X)$. Consider the following:

(DP1) $0 \leq D(A, B) \leq 1$

(DP2) $D(A, B)=0$, if and only if $A=B$;

(DP3) $D(A, B)=D(B, A)$;

(DP4) if $A \subseteq B \subseteq C$, then $D(A, B) \leq D(A, C)$, and $D(B, C) \leq$ $D(A, C)$.

Definition 5. The mapping $S:$ IFS $\times$ IFS $\rightarrow[0,1]$ is called a degree of similarity between $A \in \operatorname{IFSs}(X)$ and $B \in \operatorname{IFSs}(X)$, if $S(A, B)$ satisfies the following properties:

$(\mathrm{SP} 1) 0 \leq S(A, B) \leq 1$

(SP2) $S(A, B)=1$, if and only if $A=B$;

(SP3) $S(A, B)=S(B, A)$;

(SP4) if $A \subseteq B \subseteq C$, then $S(A, B) \geq S(A, C)$, and $S(B, C) \geq$ $S(A, C)$.

Because distance and similarity measures are complementary concepts, similarity measures can be used to define distance measures and vice versa.

\section{Existing Similarity Measures}

Let $X=\left\{x_{1}, x_{2}, \ldots, x_{n}\right\}$ be a universe of discourse. $A \in$ $\operatorname{IFSs}(X)$ and $B \in \operatorname{IFSs}(X)$ are two IFSs in $X$, denoted by $A=\left\{\left\langle x, \mu_{A}(x), v_{A}(x)\right\rangle \mid x \in X\right\}$ and $B=\left\{\left\langle x, \mu_{B}(x), v_{B}(x)\right\rangle \mid\right.$ $x \in X\}$, respectively.
Considering the outcome of the analysis presented by Bustince and Burillo [5], which concluded that the intuitionistic fuzzy sets and the vague sets are similar, Chen [29] proposed the first similarity measure for IFSs defined as

$$
\begin{aligned}
& S_{C}(A, B) \\
& \quad=1-\frac{\sum_{i=1}^{n}\left|\left(\mu_{A}\left(x_{i}\right)-v_{A}\left(x_{i}\right)\right)-\left(\mu_{B}\left(x_{i}\right)-v_{B}\left(x_{i}\right)\right)\right|}{2 n} .
\end{aligned}
$$

Hong and Kim [30] proposed the following measure to overcome the deficiency of Chen's similarity measure:

$$
\begin{aligned}
& S_{H}(A, B) \\
& \quad=1-\frac{\sum_{i=1}^{n}\left|\left(\mu_{A}\left(x_{i}\right)-\mu_{B}\left(x_{i}\right)\right)-\left(v_{A}\left(x_{i}\right)-v_{B}\left(x_{i}\right)\right)\right|}{2 n} .
\end{aligned}
$$

Following the work of Hong and $\mathrm{Kim}, \mathrm{Li}$ and $\mathrm{Xu}$ [31] proposed new similarity measures:

$$
\begin{aligned}
S_{L}(A, B) & \\
= & 1-\frac{\sum_{i=1}^{n}\left|\left(\mu_{A}\left(x_{i}\right)-v_{A}\left(x_{i}\right)\right)-\left(\mu_{B}\left(x_{i}\right)-v_{B}\left(x_{i}\right)\right)\right|}{4 n} \\
& -\frac{\sum_{i=1}^{n}\left(\left|\mu_{A}\left(x_{i}\right)-\mu_{B}\left(x_{i}\right)\right|+\left|v_{A}\left(x_{i}\right)-v_{B}\left(x_{i}\right)\right|\right)}{4 n} .
\end{aligned}
$$

Taking the membership distance and the nonmember ship distance between the two IFSs into consideration, Li et al. [32] proposed a similarity measure as follows:

$S_{\mathrm{O}}(A, B)$

$$
=1-\sqrt{\frac{\sum_{i=1}^{n}\left(\left(\mu_{A}\left(x_{i}\right)-\mu_{B}\left(x_{i}\right)\right)^{2}+\left(v_{A}\left(x_{i}\right)-v_{B}\left(x_{i}\right)\right)^{2}\right)}{2 n}} .
$$

Dengfeng and Chuntian [13] pointed out that some similarity could not deal with the similarity between IFSs well. So they defined a new similarity measure for pattern recognition:

$$
S_{\mathrm{DC}}(A, B)=1-\sqrt[p]{\frac{\sum_{i=1}^{n}\left|\varphi_{A}\left(x_{i}\right)-\varphi_{B}\left(x_{i}\right)\right|^{p}}{n}},
$$

where $\varphi_{A}\left(x_{i}\right)=\left(\mu_{A}\left(x_{i}\right)+1-v_{A}\left(x_{i}\right)\right) / 2$ and $\varphi_{B}\left(x_{i}\right)=\left(\mu_{B}\left(x_{i}\right)+\right.$ $\left.1-v_{B}\left(x_{i}\right)\right) / 2$.

Mitchell [14] found that the similarity measure $S_{\mathrm{DC}}(A, B)$ would characterize two different IFSs as identical. To overcome this drawback, he provided a more realistic strong similarity measure of the following form:

$$
S_{\mathrm{HB}}(A, B)=\frac{1}{2}\left(\rho_{\mu}(A, B)+\rho_{v}(A, B)\right),
$$

where $\rho_{\mu}(A, B)=1-\sqrt[p]{\sum_{i=1}^{n}\left|\mu_{A}\left(x_{i}\right)-\mu_{B}\left(x_{i}\right)\right|^{p} / n}, \rho_{v}(A, B)=$ $1-\sqrt[p]{\sum_{i=1}^{n}\left|v_{A}\left(x_{i}\right)-v_{B}\left(x_{i}\right)\right|^{p} / n}$. 
Liang and Shi [15] proposed the following three similarity measures:

$$
S_{e}^{p}(A, B)=1-\sqrt[p]{\frac{\sum_{i=1}^{n}\left(\phi_{\mu}\left(x_{i}\right)+\phi_{v}\left(x_{i}\right)\right)^{p}}{n}},
$$

where $\phi_{\mu}\left(x_{i}\right)=\left|\mu_{A}\left(x_{i}\right)-\mu_{B}\left(x_{i}\right)\right| / 2, \phi_{v}\left(x_{i}\right)=\mid\left(1-v_{A}\left(x_{i}\right)\right)-$ $\left(1-v_{B}\left(x_{i}\right)\right) \mid / 2$. Consider

$$
S_{s}^{p}(A, B)=1-\sqrt[p]{\frac{\sum_{i=1}^{n}\left|\psi_{s 1}\left(x_{i}\right)+\psi_{s 2}\left(x_{i}\right)\right|^{p}}{n}},
$$

where $\psi_{s 1}\left(x_{i}\right)=\left|m_{A 1}\left(x_{i}\right)-m_{B 1}\left(x_{i}\right)\right| / 2, \psi_{s 2}\left(x_{i}\right)=\mid m_{A 2}\left(x_{i}\right)-$ $m_{B 2}\left(x_{i}\right)\left|/ 2, m_{A 1}\left(x_{i}\right)=\right| \mu_{A}\left(x_{i}\right)+m_{A}\left(x_{i}\right) \mid / 2, m_{B 1}\left(x_{i}\right)=$ $\left|\mu_{B}\left(x_{i}\right)+m_{B}\left(x_{i}\right)\right| / 2, m_{A 2}\left(x_{i}\right)=\left|1-v_{A}\left(x_{i}\right)+m_{A}\left(x_{i}\right)\right| / 2$, $m_{B 2}\left(x_{i}\right)=\left|1-v_{B}\left(x_{i}\right)+m_{B}\left(x_{i}\right)\right| / 2, m_{A}\left(x_{i}\right)=\mid 1-v_{A}\left(x_{i}\right)+$ $\mu_{A}\left(x_{i}\right) \mid / 2$, and $m_{B}\left(x_{i}\right)=\left|1-v_{B}\left(x_{i}\right)+\mu_{B}\left(x_{i}\right)\right| / 2$. Consider

$$
S_{h}^{p}(A, B)=1-\sqrt[p]{\frac{\sum_{i=1}^{n}\left(\eta_{1}\left(x_{i}\right)+\eta_{2}\left(x_{i}\right)+\eta_{3}\left(x_{i}\right)\right)^{p}}{3 n}},
$$

where $\eta_{1}\left(x_{i}\right)=\phi_{\mu}\left(x_{i}\right)+\phi_{v}\left(x_{i}\right)$ (defined in $\left.S_{e}^{p}\right), \eta_{2}\left(x_{i}\right)=$ $\left|\varphi_{\mu}\left(x_{i}\right)-\varphi_{v}\left(x_{i}\right)\right|$ (defined in $\left.S_{\mathrm{DC}}\right), \eta_{3}\left(x_{i}\right)=\max \left(l_{A}\left(x_{i}\right)\right.$, $\left.l_{B}\left(x_{i}\right)\right)-\min \left(l_{A}\left(x_{i}\right), l_{B}\left(x_{i}\right)\right), l_{A}\left(x_{i}\right)=\left(1-\mu_{A}\left(x_{i}\right)-v_{A}\left(x_{i}\right)\right) / 2$, and $l_{B}\left(x_{i}\right)=\left(1-\mu_{B}\left(x_{i}\right)-v_{B}\left(x_{i}\right)\right) / 2$.

Hung and Yang [12] introduced three similarity measures based on the Hausdorff distance $d_{H}(A, B)$ :

$$
\begin{aligned}
& S_{\mathrm{HY}}^{1}(A, B)=1-d_{H}(A, B), \\
& S_{\mathrm{HY}}^{2}(A, B)=\frac{e^{-d_{H}(A, B)}-e^{-1}}{1-e^{-1}}, \\
& S_{\mathrm{HY}}^{3}(A, B)=\frac{1-d_{H}(A, B)}{1+d_{H}(A, B)},
\end{aligned}
$$

where $d_{H}(A, B)=(1 / n) \sum_{i=1}^{n} \max \left(\left|\mu_{A}\left(x_{i}\right)-\mu_{B}\left(x_{i}\right)\right|, \mid v_{A}\left(x_{i}\right)-\right.$ $\left.v_{B}\left(x_{i}\right) \mid\right)$.

Based on the concept of the cosine similarity measure for fuzzy sets, a cosine similarity measure was proposed by Ye [7] as follows:

$$
\begin{aligned}
C_{\mathrm{IFS}} & (A, B) \\
& =\frac{1}{n} \sum_{i=1}^{n} \frac{\mu_{A}\left(x_{i}\right) \mu_{B}\left(x_{i}\right)+v_{A}\left(x_{i}\right) v_{B}\left(x_{i}\right)}{\sqrt{\left(\mu_{A}\left(x_{i}\right)\right)^{2}+\left(v_{A}\left(x_{i}\right)\right)^{2}} \sqrt{\left(\mu_{B}\left(x_{i}\right)\right)^{2}+\left(v_{B}\left(x_{i}\right)\right)^{2}}} .
\end{aligned}
$$

One of the latest results on similarity measure is the biparametric similarity measure for IFS, proposed by Boran and Akay [23]. It has the following form with two parameters:

$$
\begin{gathered}
S_{t}^{p}(A, B) \\
=1-\left(\sum _ { i = 1 } ^ { n } \frac { 1 } { 2 n ( 1 + p ) } \left\{\mid t\left(\mu_{A}\left(x_{i}\right)-\mu_{B}\left(x_{i}\right)\right)\right.\right. \\
-\left.\left(v_{A}\left(x_{i}\right)-v_{B}\left(x_{i}\right)\right)\right|^{p} \\
+\mid t\left(v_{A}\left(x_{i}\right)-v_{B}\left(x_{i}\right)\right) \\
\left.\left.-\left.\left(u_{A}\left(x_{i}\right)-u_{B}\left(x_{i}\right)\right)\right|^{p}\right\}\right)^{1 / p},
\end{gathered}
$$

where $p=1,2,3, \ldots$ is the $L_{p}$ norm and $t=2,3,4, \ldots$ identifies the level of uncertainty.

\section{A New Similarity Measure}

Let $A=\left\{\left\langle x, \mu_{A}(x), v_{A}(x)\right\rangle \mid x \in X\right\}$ and $B=\left\{\left\langle x, \mu_{B}(x)\right.\right.$, $\left.\left.v_{B}(x)\right\rangle \mid x \in X\right\}$ be two IFSs in $X$. We propose a new similarity measure:

$$
\begin{aligned}
& S_{Y}(A, B) \\
& =\frac{1}{2 n} \sum_{i=1}^{n}\left(\sqrt{\mu_{A}\left(x_{i}\right) \mu_{B}\left(x_{i}\right)}\right. \\
& +2 \sqrt{v_{A}\left(x_{i}\right) v_{B}\left(x_{i}\right)}+\sqrt{\pi_{A}\left(x_{i}\right) \pi_{B}\left(x_{i}\right)} \\
& \left.+\sqrt{\left(1-v_{A}\left(x_{i}\right)\right)\left(1-v_{B}\left(x_{i}\right)\right)}\right),
\end{aligned}
$$

where $\pi_{A}\left(x_{i}\right)$ and $\pi_{B}\left(x_{i}\right)$ are, respectively, the hesitancy degree of the element $x_{i} \in X$ to the sets $A$ and $B$.

Theorem 6. $S_{Y}(A, B)$ is the similarity measure between two IFSs $A$ and $B$ in $X$.

Proof. For the sake of simplicity, IFSs $A$ and $B$ are denoted by $A=\left\{\left\langle\mu_{A}\left(x_{i}\right), v_{A}\left(x_{i}\right)\right\rangle\right\}$ and $B=\left\{\left\langle\mu_{B}\left(x_{i}\right), v_{B}\left(x_{i}\right)\right\rangle\right\}$, respectively.

(SP1) For each $x, y \in[0,+\infty]$, we have $0 \leq \sqrt{x y} \leq(x+y) / 2$.

Given $0 \leq \mu_{A}\left(x_{i}\right) \leq 1,0 \leq v_{A}\left(x_{i}\right) \leq 1,0 \leq \pi_{A}\left(x_{i}\right) \leq 1,0 \leq$ $\mu_{B}\left(x_{i}\right) \leq 1,0 \leq v_{B}\left(x_{i}\right) \leq 1,0 \leq \pi_{B}\left(x_{i}\right) \leq 1,0 \leq 1-v_{A}\left(x_{i}\right) \leq 1$, and $0 \leq 1-v_{B}\left(x_{i}\right) \leq 1$, we get

$$
\begin{aligned}
& 0 \leq \sqrt{\mu_{A}\left(x_{i}\right) \mu_{B}\left(x_{i}\right)} \leq \frac{\mu_{A}\left(x_{i}\right)+\mu_{B}\left(x_{i}\right)}{2}, \\
& 0 \leq \sqrt{v_{A}\left(x_{i}\right) v_{B}\left(x_{i}\right)} \leq \frac{v_{A}\left(x_{i}\right)+v_{B}\left(x_{i}\right)}{2},
\end{aligned}
$$




$$
\begin{gathered}
0 \leq \sqrt{\pi_{A}\left(x_{i}\right) \pi_{B}\left(x_{i}\right)} \leq \frac{\pi_{A}\left(x_{i}\right)+\pi_{B}\left(x_{i}\right)}{2}, \\
0 \leq \sqrt{\left(1-v_{A}\left(x_{i}\right)\right)\left(1-v_{B}\left(x_{i}\right)\right)} \leq \frac{1-v_{A}\left(x_{i}\right)+1-v_{B}\left(x_{i}\right)}{2} .
\end{gathered}
$$

Then we have

$$
\begin{aligned}
0 \leq & \sqrt{\mu_{A}\left(x_{i}\right) \mu_{B}\left(x_{i}\right)}+2 \sqrt{v_{A}\left(x_{i}\right) v_{B}\left(x_{i}\right)} \\
& +\sqrt{\pi_{A}\left(x_{i}\right) \pi_{B}\left(x_{i}\right)}+\sqrt{\left(1-v_{A}\left(x_{i}\right)\right)\left(1-v_{B}\left(x_{i}\right)\right)} \\
\leq & \frac{\mu_{A}\left(x_{i}\right)+\mu_{B}\left(x_{i}\right)}{2}+2 \cdot \frac{v_{A}\left(x_{i}\right)+v_{B}\left(x_{i}\right)}{2} \\
& +\frac{\pi_{A}\left(x_{i}\right)+\pi_{B}\left(x_{i}\right)}{2}+\frac{1-v_{A}\left(x_{i}\right)+1-v_{B}\left(x_{i}\right)}{2} \\
= & 1+\frac{\mu_{A}\left(x_{i}\right)+v_{A}\left(x_{i}\right)+\pi_{A}\left(x_{i}\right)}{2} \\
& +\frac{\mu_{B}\left(x_{i}\right)+v_{B}\left(x_{i}\right)+\pi_{B}\left(x_{i}\right)}{2}
\end{aligned}
$$

$=2$.

Hence,

$$
\begin{aligned}
0 \leq \sum_{i=1}^{n}( & \sqrt{\mu_{A}\left(x_{i}\right) \mu_{B}\left(x_{i}\right)}+2 \sqrt{v_{A}\left(x_{i}\right) v_{B}\left(x_{i}\right)} \\
& +\sqrt{\pi_{A}\left(x_{i}\right) \pi_{B}\left(x_{i}\right)} \\
& \left.+\sqrt{\left(1-v_{A}\left(x_{i}\right)\right)\left(1-v_{B}\left(x_{i}\right)\right)}\right) \leq 2 n .
\end{aligned}
$$

Finally, we get the following inequality: $0 \leq S_{Y}(A, B) \leq$ $(1 / 2 n) \cdot 2 n=1$.

Thus, $0 \leq S_{Y}(A, B) \leq 1$.

(SP2) We know that $\sqrt{x y}$ assumes its maximum value $(x+$ $y) / 2$ when $x=y$. Therefore, we have

$$
\begin{aligned}
S_{Y}(A, B)=1 \Longleftrightarrow & \sqrt{\mu_{A}\left(x_{i}\right) \mu_{B}\left(x_{i}\right)}+2 \sqrt{v_{A}\left(x_{i}\right) v_{B}\left(x_{i}\right)} \\
& +\sqrt{\pi_{A}\left(x_{i}\right) \pi_{B}\left(x_{i}\right)} \\
& +\sqrt{\left(1-v_{A}\left(x_{i}\right)\right)\left(1-v_{B}\left(x_{i}\right)\right)}=2 \\
\Longleftrightarrow & \mu_{A}\left(x_{i}\right) \mu_{B}\left(x_{i}\right), v_{A}\left(x_{i}\right) v_{B}\left(x_{i}\right), \\
& \pi_{A}\left(x_{i}\right) \pi_{B}\left(x_{i}\right), 1-v_{A}\left(x_{i}\right)=1-v_{B}\left(x_{i}\right) \\
\Longleftrightarrow & A=B .
\end{aligned}
$$

Thus, $S_{Y}(A, B)=1$, if and only if $A=B$.

(SP3) It is easy to note that the expression of $S_{Y}(A, B)$ is commutative. So we have

$$
S_{Y}(A, B)=S_{Y}(B, A) .
$$

(SP4) Let $C$ be another IFS in $X$, denoted by $C=$ $\left\{\left\langle\mu_{C}\left(x_{i}\right), v_{C}\left(x_{i}\right)\right\rangle\right\} . A, B$, and $C$ satisfy the relation $A \subseteq$ $B \subseteq C$. Then, we have $0 \leq \mu_{A}\left(x_{i}\right) \leq \mu_{B}\left(x_{i}\right) \leq \mu_{C}\left(x_{i}\right) \leq$ 1 and $0 \leq v_{C}\left(x_{i}\right) \leq v_{B}\left(x_{i}\right) \leq v_{A}\left(x_{i}\right) \leq 1$, for all $x \in X$. Based on (17), the similarity measures between $(B, C)$ and $(A, C)$ can be written as

$$
\begin{aligned}
& S_{Y}(B, C)=\frac{1}{2 n} \sum_{i=1}^{n}\left(\sqrt{\mu_{B}\left(x_{i}\right) \mu_{C}\left(x_{i}\right)}+\sqrt{v_{B}\left(x_{i}\right) v_{C}\left(x_{i}\right)}\right. \\
& +\sqrt{\pi_{B}\left(x_{i}\right) \pi_{C}\left(x_{i}\right)} \\
& \left.+\sqrt{\left(1-v_{B}\left(x_{i}\right)\right)\left(1-v_{C}\left(x_{i}\right)\right)}\right), \\
& \begin{aligned}
S_{Y}(A, C)=\frac{1}{2 n} \sum_{i=1}^{n}( & \sqrt{\mu_{A}\left(x_{i}\right) \mu_{C}\left(x_{i}\right)}+\sqrt{v_{A}\left(x_{i}\right) v_{C}\left(x_{i}\right)} \\
& +\sqrt{\pi_{A}\left(x_{i}\right) \pi_{C}\left(x_{i}\right)} \\
& \left.+\sqrt{\left(1-v_{A}\left(x_{i}\right)\right)\left(1-v_{C}\left(x_{i}\right)\right)}\right) .
\end{aligned}
\end{aligned}
$$

For $a, b \in[0,1], a+b \leq 1$ we can define a function $f$ as

$$
\begin{aligned}
f(x, y)= & \sqrt{a x}+2 \sqrt{b y}+\sqrt{(1-a-b)(1-x-y)} \\
& +\sqrt{(1-b)(1-y)},
\end{aligned}
$$

where $x, y \in[0,1], x+y \in[0,1]$.

Then we have

$$
\begin{gathered}
\frac{\partial f}{\partial x}=\frac{\sqrt{a}}{2 \sqrt{x}}-\frac{\sqrt{1-a-b}}{2 \sqrt{1-x-y}}, \\
\frac{\partial f}{\partial y}=\frac{\sqrt{b}}{\sqrt{y}}-\frac{\sqrt{1-a-b}}{2 \sqrt{1-x-y}}-\frac{\sqrt{1-b}}{2 \sqrt{1-y}} .
\end{gathered}
$$

Given $a \leq x \leq 1, b \leq 1$, we have

$$
\begin{aligned}
& \left.\frac{\partial f}{\partial x}\right|_{y=b} \\
& \quad=\frac{\sqrt{a}}{2 \sqrt{x}}-\frac{\sqrt{1-a-b}}{2 \sqrt{1-x-y}} \\
& \quad=\frac{(a-x)(1-b)}{2 \sqrt{x(1-x-y)}(\sqrt{a(1-x-y)}+\sqrt{(1-a-b) x})} \\
& \quad \leq 0,
\end{aligned}
$$

which means that $f$ is a decreasing function of $x$, when $y=b$, $x \geq a$.

For $0 \leq x \leq a, b \leq 1$, we can get $\left.(\partial f / \partial x)\right|_{y=b} \geq 0$, which means that $f$ is an increasing function of $x$, when $y=b, x \leq$ a. 
Similarly, as for $\partial f / \partial y$, we can get

$$
\begin{aligned}
\left.\frac{\partial f}{\partial y}\right|_{x=a} & \\
= & \frac{(b-y)(1-a)}{2 \sqrt{y(1-x-y)}(\sqrt{b(1-x-y)}+\sqrt{(1-a-b) y})} \\
& +\frac{b-y}{2 \sqrt{y(1-y)}(\sqrt{b(1-y)}+\sqrt{(1-b) y})}
\end{aligned}
$$

which reveals that $f$ is an increasing function of $y$ for $x=a$, $y \leq b$, but a decreasing function when $x=a, y \geq b$.

Given $a=\mu_{A}\left(x_{i}\right), b=v_{A}\left(x_{i}\right)$, and two couples $\left(\mu_{B}\left(x_{i}\right)\right.$, $\left.v_{B}\left(x_{i}\right)\right),\left(\mu_{C}\left(x_{i}\right), v_{C}\left(x_{i}\right)\right)$, satisfying $a=\mu_{A}\left(x_{i}\right) \leq \mu_{B}\left(x_{i}\right) \leq$ $\mu_{C}\left(x_{i}\right), v_{C}\left(x_{i}\right) \leq v_{B}\left(x_{i}\right) \leq v_{A}\left(x_{i}\right)=b$, we can get

$$
\begin{gathered}
f\left(\mu_{C}\left(x_{i}\right), b\right) \leq f\left(\mu_{B}\left(x_{i}\right), b\right) \leq f(a, b), \\
f\left(a, v_{C}\left(x_{i}\right)\right) \leq f\left(a, v_{B}\left(x_{i}\right)\right) \leq f(a, b) .
\end{gathered}
$$

Since $f(x, y)$ is a continuous concave function, it is true that $f\left(\mu_{C}\left(x_{i}\right), v_{C}\left(x_{i}\right)\right) \leq f\left(\mu_{B}\left(x_{i}\right), v_{B}\left(x_{i}\right)\right)$; that is,

$$
\begin{aligned}
& \sqrt{\mu_{A}\left(x_{i}\right) \mu_{C}\left(x_{i}\right)}+\sqrt{v_{A}\left(x_{i}\right) v_{C}\left(x_{i}\right)}+\sqrt{\pi_{A}\left(x_{i}\right) \pi_{C}\left(x_{i}\right)} \\
& +\sqrt{\left(1-v_{A}\left(x_{i}\right)\right)\left(1-v_{C}\left(x_{i}\right)\right)} \\
& \leq \sqrt{\mu_{A}\left(x_{i}\right) \mu_{B}\left(x_{i}\right)}+2 \sqrt{v_{A}\left(x_{i}\right) v_{B}\left(x_{i}\right)} \\
& +\sqrt{\pi_{A}\left(x_{i}\right) \pi_{B}\left(x_{i}\right)}+\sqrt{\left(1-v_{A}\left(x_{i}\right)\right)\left(1-v_{B}\left(x_{i}\right)\right)},
\end{aligned}
$$

which indicates that $S_{Y}(A, B) \geq S_{Y}(A, C)$.

Similarly, if we suppose $a=\mu_{C}\left(x_{i}\right) b=v_{C}\left(x_{i}\right)$, considering another two couples $\left(\mu_{A}\left(x_{i}\right), v_{A}\left(x_{i}\right)\right),\left(\mu_{B}\left(x_{i}\right), v_{B}\left(x_{i}\right)\right)$, where $\mu_{A}\left(x_{i}\right) \leq \mu_{B}\left(x_{i}\right) \leq \mu_{C}\left(x_{i}\right)=a, b=v_{C}\left(x_{i}\right) \leq v_{B}\left(x_{i}\right) \leq$ $v_{A}\left(x_{i}\right)$, we have

$$
\begin{gathered}
f\left(\mu_{A}\left(x_{i}\right), b\right) \leq f\left(\mu_{B}\left(x_{i}\right), b\right) \leq f(a, b), \\
f\left(a, v_{A}\left(x_{i}\right)\right) \leq f\left(a, \mu_{B}\left(x_{i}\right)\right) \leq f(a, b) .
\end{gathered}
$$

Considering the continuity and concavity of $f(x, y)$, we can finally get $f\left(\mu_{A}\left(x_{i}\right), v_{A}\left(x_{i}\right)\right) \leq f\left(\mu_{B}\left(x_{i}\right), v_{B}\left(x_{i}\right)\right)$, also written as

$$
\begin{aligned}
& \sqrt{\mu_{A}\left(x_{i}\right) \mu_{C}\left(x_{i}\right)}+\sqrt{v_{A}\left(x_{i}\right) v_{C}\left(x_{i}\right)}+\sqrt{\pi_{A}\left(x_{i}\right) \pi_{C}\left(x_{i}\right)} \\
& +\sqrt{\left(1-v_{A}\left(x_{i}\right)\right)\left(1-v_{C}\left(x_{i}\right)\right)} \\
& \leq \sqrt{\mu_{B}\left(x_{i}\right) \mu_{C}\left(x_{i}\right)}+2 \sqrt{v_{B}\left(x_{i}\right) v_{C}\left(x_{i}\right)} \\
& +\sqrt{\pi_{B}\left(x_{i}\right) \pi_{C}\left(x_{i}\right)}+\sqrt{\left(1-v_{B}\left(x_{i}\right)\right)\left(1-v_{C}\left(x_{i}\right)\right)} .
\end{aligned}
$$

So we have $S_{Y}(B, C) \geq S_{Y}(A, C)$.

Thus, $S_{Y}(A, B)$ satisfies (SP4).

So the similarity measure $S_{Y}(A, B)$ satisfies all properties in Definition 5. It is a similarity measure between IFSs.

Considering the weights of $x_{i}$, we can define the weighted similarity between two IFSs as follows:

$$
\begin{aligned}
S_{\mathrm{WY}}(A, B)=\frac{1}{2} \sum_{i=1}^{n} w_{i} & \left(\sqrt{\mu_{A}\left(x_{i}\right) \mu_{B}\left(x_{i}\right)}\right. \\
& +2 \sqrt{v_{A}\left(x_{i}\right) v_{B}\left(x_{i}\right)}+\sqrt{\pi_{A}\left(x_{i}\right) \pi_{B}\left(x_{i}\right)} \\
& \left.+\sqrt{\left(1-v_{A}\left(x_{i}\right)\right)\left(1-v_{B}\left(x_{i}\right)\right)}\right),
\end{aligned}
$$

where $w_{i}$ is the weights factor of the features $x_{i}, w_{i} \in[0,1]$ and $\sum_{i=1}^{n} w_{i}=1$.

Theorem 7. $S_{W Y}(A, B)$ is the similarity measure between two IFSs $A$ and $B$ in $X$.

Proof. (SP1) From the proof of (SP1) in the last theorem, we can get

$$
\begin{aligned}
0 \leq \sum_{i=1}^{n} w_{i}( & \sqrt{\mu_{A}\left(x_{i}\right) \mu_{B}\left(x_{i}\right)}+2 \sqrt{v_{A}\left(x_{i}\right) v_{B}\left(x_{i}\right)} \\
& +\sqrt{\pi_{A}\left(x_{i}\right) \pi_{B}\left(x_{i}\right)} \\
& \left.+\sqrt{\left(1-v_{A}\left(x_{i}\right)\right)\left(1-v_{B}\left(x_{i}\right)\right)}\right) \\
\leq \sum_{i=1}^{n} 2 w_{i} & =2 \cdot \sum_{i=1}^{n} w_{i}=2 .
\end{aligned}
$$

Therefore, $0 \leq S_{\mathrm{WY}}(A, B) \leq 1$.

(SP2) Considering the implication rule in the proof of (SP2) for Theorem 6, we have

$$
\begin{aligned}
S_{\mathrm{WY}}(A, B)=1 \Longleftrightarrow w_{i}( & \sqrt{\mu_{A}\left(x_{i}\right) \mu_{B}\left(x_{i}\right)} \\
& +2 \sqrt{v_{A}\left(x_{i}\right) v_{B}\left(x_{i}\right)} \\
& +\sqrt{\pi_{A}\left(x_{i}\right) \pi_{B}\left(x_{i}\right)} \\
& \left.+\sqrt{\left(1-v_{A}\left(x_{i}\right)\right)\left(1-v_{B}\left(x_{i}\right)\right)}\right) \\
= & 2 w_{i}
\end{aligned}
$$




$$
\begin{aligned}
\Longleftrightarrow & \sqrt{\mu_{A}\left(x_{i}\right) \mu_{B}\left(x_{i}\right)}+2 \sqrt{v_{A}\left(x_{i}\right) v_{B}\left(x_{i}\right)} \\
& +\sqrt{\pi_{A}\left(x_{i}\right) \pi_{B}\left(x_{i}\right)} \\
& +\sqrt{\left(1-v_{A}\left(x_{i}\right)\right)\left(1-v_{B}\left(x_{i}\right)\right)}=2 \\
\Longleftrightarrow & \mu_{A}\left(x_{i}\right) \mu_{B}\left(x_{i}\right), v_{A}\left(x_{i}\right) v_{B}\left(x_{i}\right), \\
& \pi_{A}\left(x_{i}\right) \pi_{B}\left(x_{i}\right), 1-v_{A}\left(x_{i}\right)=1-v_{B}\left(x_{i}\right) \\
\Longleftrightarrow & A=B .
\end{aligned}
$$

So we get $S_{\mathrm{WY}}(A, B)=1 \Leftrightarrow A=B$.

(SP3)It is straightforward that $S_{\mathrm{WY}}(A, B)$ satisfies (SP3).

(SP4) Since all $w_{i} \geq 0$, we can get

$$
\begin{aligned}
& w_{i}\left(\sqrt{\mu_{A}\left(x_{i}\right) \mu_{C}\left(x_{i}\right)}+\sqrt{v_{A}\left(x_{i}\right) v_{C}\left(x_{i}\right)}\right. \\
& \left.+\sqrt{\pi_{A}\left(x_{i}\right) \pi_{C}\left(x_{i}\right)}+\sqrt{\left(1-v_{A}\left(x_{i}\right)\right)\left(1-v_{C}\left(x_{i}\right)\right)}\right) \\
& \leq w_{i}\left(\sqrt{\mu_{A}\left(x_{i}\right) \mu_{B}\left(x_{i}\right)}+2 \sqrt{v_{A}\left(x_{i}\right) v_{B}\left(x_{i}\right)}\right. \\
& \left.+\sqrt{\pi_{A}\left(x_{i}\right) \pi_{B}\left(x_{i}\right)}+\sqrt{\left(1-v_{A}\left(x_{i}\right)\right)\left(1-v_{B}\left(x_{i}\right)\right)}\right), \\
& w_{i}\left(\sqrt{\mu_{A}\left(x_{i}\right) \mu_{C}\left(x_{i}\right)}+\sqrt{v_{A}\left(x_{i}\right) v_{C}\left(x_{i}\right)}\right. \\
& \left.+\sqrt{\pi_{A}\left(x_{i}\right) \pi_{C}\left(x_{i}\right)}+\sqrt{\left(1-v_{A}\left(x_{i}\right)\right)\left(1-v_{C}\left(x_{i}\right)\right)}\right) \\
& \leq w_{i}\left(\sqrt{\mu_{B}\left(x_{i}\right) \mu_{C}\left(x_{i}\right)}+2 \sqrt{v_{B}\left(x_{i}\right) v_{C}\left(x_{i}\right)}\right. \\
& \left.+\sqrt{\pi_{B}\left(x_{i}\right) \pi_{C}\left(x_{i}\right)}+\sqrt{\left(1-v_{B}\left(x_{i}\right)\right)\left(1-v_{C}\left(x_{i}\right)\right)}\right) .
\end{aligned}
$$

Finally we have $S_{\mathrm{WY}}(A, B) \geq S_{\mathrm{WY}}(A, C), S_{\mathrm{WY}}(B, C) \geq$ $S_{\mathrm{WY}}(A, C)$.

So far, we have finished the proof that $S_{\mathrm{WY}}(A, B)$ is a similarity measure between IFSs $A$ and $B$.

\section{Numerical Comparisons}

In order to illustrate the superiority of the proposed similarity measure, a comparison between the proposed similarity measure and all the existing similarity measures is conducted based on the numerical cases in [23]. Table 1 presents a comprehensive comparison of the similarity measures for IFS with counterintuitive examples $\left(p=1\right.$ for $S_{\mathrm{HB}}, S_{e}^{p}, S_{s}^{p}, S_{h}^{p}$ and $p=1, t=2$ for $S_{t}^{p}$ ).

We can see that $S_{C}(A, B)=S_{\mathrm{DC}}(A, B)=C_{\mathrm{IFS}}(A, B)=1$ for two different IFSs $A=\langle 0.3,0.3\rangle$ and $B=\langle 0.4,0.4\rangle$. This indicates that the second axiom of similarity measure (SP2) is not satisfied by $S_{C}(A, B), S_{\mathrm{DC}}(A, B)$, and $C_{\mathrm{IFS}}(A, B)$. This also can be illustrated by $S_{C}(A, B)=S_{\mathrm{DC}}(A, B)=1$ when $A=\langle 0.5,0.5\rangle, B=\langle 0,0\rangle$ and $A=\langle 0.4,0.2\rangle, B=\langle 0.5,0.3\rangle$.
As for $S_{H}, S_{\mathrm{O}}, S_{\mathrm{HB}}, S_{e}^{p}, S_{s}^{p}$, and $S_{h}^{p}$, different pairs of $A, B$ may provide the identical results, which cannot satisfy the application of pattern recognition. It can be read from Table 1 that $S_{\mathrm{HB}}=0.9$ for both $A=\langle 0.3,0.3\rangle, B=\langle 0.4,0.4\rangle$ and $A=\langle 0.3,0.4\rangle, B=\langle 0.4,0.3\rangle$. Such situation seems to be going from bad to worse for $S_{\mathrm{HY}}^{1}$, where all the cases take the same similarity degree except Case 3 and Case 4. $S_{\mathrm{HY}}^{2}$ and $S_{\mathrm{HY}}^{3}$ are also stuck with this problem. $S_{t}^{p}$ seems to be reasonable without any counterintuitive results. However, we can notice an interesting situation when comparing Case 3 and Case 4. Suppose two vote results, "all in favor" and "all abstentions," which, respectively, represent $A=\langle 1,0\rangle$ and $B=\langle 0,0\rangle$. It is reasonable to take the similarity degree between them as 0.5. $A^{\prime}=\langle 0.5,0.5\rangle$ and $B^{\prime}=\langle 0,0\rangle$ can be interpreted as "the vote for resolution is half in favor, half against" and "the vote for resolution is all abstentions," respectively. The similarity degree between them is 0.833 , which is greater than 0.5 . From the view of vote practice, the similarity degree between $A$ and $B$ should be equal to or not less than the similarity between $A^{\prime}$ and $B^{\prime}$. Furthermore, it is worth considering the determination of parameters $p$ and $t$ in $S_{t}^{p}$. In such a sense, the proposed similarity measure is the most reasonable one with a relative simple expression and has none of the counterintuitive cases.

In order to study the effectiveness of the proposed similarity measure for IFS in the application of pattern recognition, we consider the pattern recognition problem discussed in $[7,13]$.

Suppose there are $m$ patterns, which can be represented by IFSs $A_{j}=\left\{\left\langle x_{i}, \mu_{A_{j}}\left(x_{i}\right), v_{A_{j}}\left(x_{i}\right)\right\rangle \mid x_{i} \in X\right\}, A_{j} \in$ $\operatorname{IFSs}(X), j=1,2, \ldots, m$. Let the sample to be recognized be denoted by $B=\left\{\left\langle x_{i}, \mu_{B}\left(x_{i}\right), v_{B}\left(x_{i}\right)\right\rangle \mid x_{i} \in X\right\}$. According to the recognition principle of maximum degree of similarity between IFSs, the process of assigning $B$ to $A_{k}$ is described by [7]:

$$
k=\arg \max _{j=1,2, \ldots, m}\left\{S\left(A_{j}, B\right)\right\}
$$

Example 8. Assume that there exist three known patterns $A_{1}$, $A_{2}$, and $A_{3}$, with class labels $C_{1}, C_{2}$, and $C_{3}$, respectively. Each pattern can be expressed by IFS in $X=\left\{x_{1}, x_{2}, x_{3}\right\}$ as follows:

$$
\begin{aligned}
& A_{1}=\left\{\left\langle x_{1}, 1,0\right\rangle,\left\langle x_{2}, 0.8,0\right\rangle,\left\langle x_{3}, 0.7,0.1\right\rangle\right\}, \\
& A_{2}=\left\{\left\langle x_{1}, 0.8,0.1\right\rangle,\left\langle x_{2}, 1,0\right\rangle,\left\langle x_{3}, 0.9,0\right\rangle\right\}, \\
& A_{3}=\left\{\left\langle x_{1}, 0.6,0.2\right\rangle,\left\langle x_{2}, 0.8,0\right\rangle,\left\langle x_{3}, 1,0\right\rangle\right\} .
\end{aligned}
$$

The sample $B$ which needs to be recognized is as follows:

$$
B=\left\{\left\langle x_{1}, 0.5,0.3\right\rangle,\left\langle x_{2}, 0.6,0.2\right\rangle,\left\langle x_{3}, 0.8,0.1\right\rangle\right\} .
$$

The similarity degree between $A_{i}(i=1,2,3)$ and $B$ calculated by (17) is

$$
\begin{gathered}
S_{Y}\left(A_{1}, B\right)=0.887, \\
S_{Y}\left(A_{2}, B\right)=0.913, \\
S_{Y}\left(A_{3}, B\right)=0.936 .
\end{gathered}
$$


TABLE 1: The comparison of similarity measures (counterintuitive cases are in bold type).

\begin{tabular}{|c|c|c|c|c|c|c|}
\hline & 1 & 2 & 3 & 4 & 5 & 6 \\
\hline$A$ & $\langle 0.3,0.3\rangle$ & $\langle 0.3,0.4\rangle$ & $\langle 1,0\rangle$ & $\langle 0.5,0.5\rangle$ & $\langle 0.4,0.2\rangle$ & $\langle 0.4,0.2\rangle$ \\
\hline B & $\langle 0.4,0.4\rangle$ & $\langle 0.4,0.3\rangle$ & $\langle 0,0\rangle$ & $\langle 0,0\rangle$ & $\langle 0.5,0.3\rangle$ & $\langle 0.5,0.2\rangle$ \\
\hline$S_{C}$ & 1 & 0.9 & 0.5 & 1 & 1 & 0.95 \\
\hline$S_{H}$ & 0.9 & 0.9 & 0.5 & 0.5 & 0.9 & 0.95 \\
\hline$S_{L}$ & 0.95 & 0.9 & 0.5 & 0.75 & 0.95 & 0.95 \\
\hline$S_{O}$ & 0.9 & 0.9 & 0.3 & 0.5 & 0.9 & 0.93 \\
\hline$S_{\mathrm{DC}}$ & 1 & 0.9 & 0.5 & 1 & 1 & 0.95 \\
\hline$S_{\mathrm{HB}}$ & 0.9 & 0.9 & 0.5 & 0.5 & 0.9 & 0.95 \\
\hline$S_{e}^{p}$ & 0.9 & 0.9 & 0.5 & 0.5 & 0.9 & 0.95 \\
\hline$S_{s}^{p}$ & 0.95 & 0.9 & 0.5 & 0.75 & 0.95 & 0.95 \\
\hline$S_{h}^{p}$ & 0.933 & 0.933 & 0.5 & 0.67 & 0.933 & 0.95 \\
\hline$S_{\mathrm{HY}}^{1}$ & 0.9 & 0.9 & 0 & 0.5 & 0.9 & 0.9 \\
\hline$S_{\mathrm{HY}}^{2}$ & 0.85 & 0.85 & 0 & 0.38 & 0.85 & 0.85 \\
\hline$S_{\mathrm{HY}}^{3}$ & 0.82 & 0.82 & 0 & 0.33 & 0.82 & 0.82 \\
\hline$C_{\mathrm{IFS}}$ & 1 & 0.96 & 0 & 0 & 0.9971 & 0.9965 \\
\hline$S_{t}^{p}$ & 0.967 & 0.9 & 0.5 & 0.833 & 0.937 & 0.95 \\
\hline$S_{Y}$ & 0.985 & 0.994 & 0.5 & 0.354 & 0.936 & 0.896 \\
\hline
\end{tabular}

It can be observed that pattern $B$ should be classified to $A_{3}$ with a class label $C_{3}$. According to the recognition principle of maximum degree of similarity between IFSs, this result is in agreement with the one obtained in $[7,13]$.

Let us assume that the weights of $x_{1}, x_{2}$, and $x_{3}$ are $0.5,0.3$, and 0.2 , respectively, as they were assumed in [7]. Considering (32), we can get

$$
\begin{aligned}
& S_{\mathrm{WY}}\left(A_{1}, B\right)=0.853, \\
& S_{\mathrm{WY}}\left(A_{2}, B\right)=0.919, \\
& S_{\mathrm{WY}}\left(A_{3}, B\right)=0.949 .
\end{aligned}
$$

According to (36), $B$ can be recognized as $A_{3}$, which is identical to the result obtained in $[7,13]$.

To make our similarity measure more transparent and comparable with the measures proposed earlier by other authors, the example analyzed in [17] will be discussed next.

Example 9. Assume that there are three IFS patterns in $X=$ $\left\{x_{1}, x_{2}, x_{3}\right\}$. The three patterns are denoted as follows:

$$
\begin{aligned}
& A_{1}=\left\{\left\langle x_{1}, 0.3,0.3\right\rangle,\left\langle x_{2}, 0.2,0.2\right\rangle,\left\langle x_{3}, 0.1,0.1\right\rangle\right\}, \\
& A_{2}=\left\{\left\langle x_{1}, 0.2,0.2\right\rangle,\left\langle x_{2}, 0.2,0.2\right\rangle,\left\langle x_{3}, 0.2,0.2\right\rangle\right\}, \\
& A_{3}=\left\{\left\langle x_{1}, 0.4,0.4\right\rangle,\left\langle x_{2}, 0.4,0.4\right\rangle,\left\langle x_{3}, 0.4,0.4\right\rangle\right\} .
\end{aligned}
$$

Assume that a sample $B=\left\{\left\langle x_{1}, 0.3,0.3\right\rangle,\left\langle x_{2}, 0.2,0.2\right\rangle\right.$, $\left.\left\langle x_{3}, 0.1,0.1\right\rangle\right\}$ is to be classified.

The similarity degrees of $S\left(A_{1}, B\right), S\left(A_{2}, B\right)$, and $S\left(A_{3}, B\right)$ are calculated by (5)-(16) for all existing similarity measures and shown in Table 2 , where $p=1$ for $S_{\mathrm{HB}}, S_{e}^{p}, S_{s}^{p}, S_{h}^{p}$ and $p=1, t=2$ for $S_{t}^{p}$.
The proposed similarity measure $S_{Y}$ can be calculated by (17) as follows:

$$
\begin{gathered}
S_{Y}\left(A_{1}, B\right)=1, \\
S_{Y}\left(A_{2}, B\right)=0.990, \\
S_{Y}\left(A_{3}, B\right)=0.932 .
\end{gathered}
$$

It is obvious that $B$ is equal to $A_{1}$. This indicates that sample $B$ should be classified to $A_{1}$. However, the similarity degrees of $S\left(A_{1}, B\right), S\left(A_{2}, B\right)$, and $S\left(A_{3}, B\right)$ are equal to each other when $S_{C}, S_{H}, S_{\mathrm{DC}}$, and $C_{\mathrm{IFS}}$ are employed. These four similarity measures are not capable of discriminating difference between the three patterns. Fortunately, the results of $S_{Y}\left(A_{i}, B\right)(i=1,2,3)$ can be used to make correct classification conclusion. This means that the proposed similarity measure shows an identical performance with majority of the existing measures.

\section{Applications in Pattern Recognition}

Along with the previous investigation of classification capabilities of the proposed measure, an additional experiment discussed in $[7,22,23,33-36]$, will be presented as an application in pattern recognition. In this paper, we propose an alternative approach to medical diagnosis using the newly defined similarity measure.

Let us consider the same data as in $[7,22,23,33-$ 36]. Suppose that there are four patients Al, Bob, Joe, Ted, represented as $P=\{\mathrm{Al}, \mathrm{Bob}, \mathrm{Joe}, \mathrm{Ted}\}$. Their symptoms are $S=\{$ Temperature, Headache, Stomach pain, Cough, Chest pain\}. The set of diagnoses is defined as $D=\{$ Viral fever, Malaria, Typhoid, Stomach problem, Chest problem\}. The intuitionistic fuzzy relation $P \rightarrow S$ is presented in Table 3 . Table 4 gives the intuitionistic fuzzy relation $S \rightarrow D$. Each element of the tables is given in the form of IFV, which is 
TABLE 2: The similarity measures between the known patterns and the unknown patterns in Example 9 (patterns not discriminated are in bold type).

\begin{tabular}{lcccccc}
\hline & $S\left(A_{1}, B\right)$ & $S\left(A_{2}, B\right)$ & $S\left(A_{3}, B\right)$ & & $S\left(A_{1}, B\right)$ & $S\left(A_{2}, B\right)$ \\
\hline$S_{\mathrm{C}}$ & $\mathbf{1}$ & $\mathbf{1}$ & $\mathbf{1}$ & $S_{s}^{p}$ & 1 & 0.967 \\
$S_{H}$ & $\mathbf{1}$ & $\mathbf{1}$ & $\mathbf{1}$ & $S_{h}^{p}$ & 1 & 0.900 \\
$S_{L}$ & 1 & 0.967 & 0.9 & $S_{\mathrm{HY}}^{1}$ & 1 & 0.956 \\
$S_{\mathrm{O}}$ & 1 & 0.918 & 0.784 & $S_{\mathrm{HY}}^{2}$ & 1 & 0.967 \\
$S_{\mathrm{DC}}$ & $\mathbf{1}$ & $\mathbf{1}$ & $\mathbf{1}$ & $S_{\mathrm{HY}}^{3}$ & 1 & 0.898 \\
$S_{\mathrm{HB}}$ & 1 & 0.933 & 0.8 & $C_{\mathrm{IFS}}$ & $\mathbf{1}$ & 0.875 \\
$S_{e}^{p}$ & 1 & 0.933 & 0.8 & $S_{t}^{p}$ & 1 & $\mathbf{1}$ \\
\hline
\end{tabular}

TABLE 3: Symptoms characteristic for the patients.

\begin{tabular}{lccccc}
\hline & Temperature & Headache & Stomach pain & Cough & $(0.6,0.1)$ \\
Al & $(0.8,0.1)$ & $(0.6,0.1)$ & $(0.2,0.8)$ & $(0.1,0.7)$ & $(0.1,0.6)$ \\
Bob & $(0,0.8)$ & $(0.4,0.4)$ & $(0.6,0.1)$ & $(0.2,0.7)$ & $(0.0,0.5)$ \\
Joe & $(0.8,0.1)$ & $(0.8,0.1)$ & $(0.0,0.6)$ & $(0.7,0.2)$ & $(0.3,0.4)$ \\
Ted & $(0.6,0.1)$ & $(0.5,0.4)$ & $(0.3,0.4)$ & \\
\hline
\end{tabular}

TABLE 4: Symptoms characteristic for the diagnoses.

\begin{tabular}{lccccc}
\hline & Viral fever & Malaria & Typhoid & Stomach problem & Chest pain problem \\
\hline Temperature & $(0.4,0.0)$ & $(0.7,0.0)$ & $(0.3,0.3)$ & $(0.1,0.7)$ & $(0.1,0.8)$ \\
Headache & $(0.3,0.5)$ & $(0.2,0.6)$ & $(0.6,0.1)$ & $(0.2,0.4)$ & $(0,0.8)$ \\
Stomach pain & $(0.1,0.7)$ & $(0.0,0.9)$ & $(0.2,0.7)$ & $(0.8,0.0)$ & $(0.2,0.8)$ \\
Cough & $(0.4,0.3)$ & $(0.7,0.0)$ & $(0.2,0.6)$ & $(0.2,0.7)$ & $(0.2,0.8)$ \\
Chest pain & $(0.1,0.7)$ & $(0.1,0.8)$ & $(0.1,0.9)$ & $(0.2,0.7)$ & $(0.8,0.1)$ \\
\hline
\end{tabular}

TABLE 5: The proposed similarity measure $S_{Y}$ between each patient's symptoms and the considered set of possible diagnoses.

\begin{tabular}{lccccc}
\hline & Viral fever & Malaria & Typhoid & Stomach problem & Chest problem \\
\hline Al & $\mathbf{0 . 9 3 4 7}$ & 0.9228 & 0.9223 & 0.7673 & 0.7490 \\
Bob & 0.8124 & 0.6775 & 0.8997 & $\mathbf{0 . 9 7 6 0}$ & 0.8211 \\
Joe & 0.9152 & 0.8271 & $\mathbf{0 . 9 1 8 8}$ & 0.7917 & 0.7456 \\
Ted & $\mathbf{0 . 9 5 7 6}$ & 0.9034 & 0.9060 & 0.8577 & 0.8122 \\
\hline
\end{tabular}

a pair of numbers corresponding to the membership and nonmembership values, respectively.

In order to make a proper diagnosis for each patient, we calculate the similarity degree between each patient and each diagnose. According to the principle of maximum similarity degree, the higher similarity degree indicates a proper diagnosis. In Table 5, the similarity degree $S_{Y}$ between patients and diagnoses is presented. According to the similarity degrees in Table 5, conclusion can be made that $\mathrm{Al}$ suffers from Viral fever, Bob suffers from Stomach problem, Joe suffers from Typhoid, and Ted suffers from Viral fever. The diagnosis results for this case obtained in previous study have been presented in [23]. It is clear that our proposed method provides the same results obtained by Vlachos and Sergiadis in [33], Own in [34], and Boran and Akay in [23]. Moreover, our proposed similarity measure is calculated based on the IFNs, without any other parameters such as $p, t$ in [23]. So it can reduce the computation complexity.

\section{Conclusion}

Since most of the existing similarity measures for IFSs have provided counterintuitive results, a new similarity measure and weighted similarity measure between IFSs were proposed in this paper. The new similarity measure is calculated based on the membership degree $\mu_{A}(x)$, nonmembership degree $v_{A}(x)$, hesitancy degree $\pi_{A}(x)$, and the upper bound of membership $1-v_{A}(x)$. In some special cases where some of the existing similarity measures cannot provide reasonable results, the proposed similarity measure shows great capacity for discriminating IFSs. Moreover, investigation of the new measure's classification capability was carried out based on two numerical examples and medical diagnosis. It has been illustrated that the proposed similarity measure performs as well as or better than previous measures. Further research will be focused on its applications in other practical fields. 


\section{Conflict of Interests}

The authors declare that there is no conflict of interests regarding the publication of this paper.

\section{Acknowledgment}

This work is supported by the National Natural Science Foundation of China (nos. 61273275 and 60975026).

\section{References}

[1] L. A. Zadeh, "Fuzzy sets," Information and Control, vol. 8, pp. 338-353, 1965.

[2] K. T. Atanassov, "Intuitionistic fuzzy sets," Fuzzy Sets and Systems, vol. 20, no. 1, pp. 87-96, 1986.

[3] Z. Xu and R. R. Yager, "Dynamic intuitionistic fuzzy multiattribute decison making," International Journal of Approximate Reasoning, vol. 48, no. 1, pp. 246-262, 2008.

[4] W. Gau and D. J. Buehrer, "Vague sets," IEEE Transactions on Systems, Man and Cybernetics, vol. 23, no. 2, pp. 610-614, 1993.

[5] H. Bustince and P. Burillo, "Vague sets are intuitionistic fuzzy sets," Fuzzy Sets and Systems, vol. 79, no. 3, pp. 403-405, 1996.

[6] M. Xia and Z. Xu, "Some new similarity measures for intuitionistic fuzzy values and their application in group decision making," Journal of Systems Science and Systems Engineering, vol. 19, no. 4, pp. 430-452, 2010.

[7] J. Ye, "Cosine similarity measures for intuitionistic fuzzy sets and their applications," Mathematical and Computer Modelling, vol. 53, no. 1-2, pp. 91-97, 2011.

[8] E. Szmidt and J. Kacprzyk, "Distances between intuitionistic fuzzy sets," Fuzzy Sets and Systems, vol. 114, no. 3, pp. 505-518, 2000.

[9] W. Wang and X. Xin, "Distance measure between intuitionistic fuzzy sets," Pattern Recognition Letters, vol. 26, no. 13, pp. 20632069, 2005.

[10] P. Grzegorzewski, "Distances between intuitionistic fuzzy sets and/or interval-valued fuzzy sets based on the Hausdorff metric," Fuzzy Sets and Systems, vol. 148, no. 2, pp. 319-328, 2004.

[11] T.-Y. Chen, "A note on distances between intuitionistic fuzzy sets and/or interval-valued fuzzy sets based on the Hausdorff metric," Fuzzy Sets and Systems, vol. 158, no. 22, pp. 2523-2525, 2007.

[12] W. L. Hung and M. S. Yang, "Similarity measures of intuitionistic fuzzy sets based on Hausdorff distance," Pattern Recognition Letters, vol. 25, no. 14, pp. 1603-1611, 2004.

[13] L. Dengfeng and C. Chuntian, "New similarity measures of intuitionistic fuzzy sets and application to pattern recognitions," Pattern Recognition Letters, vol. 23, no. 1-3, pp. 221-225, 2002.

[14] H. B. Mitchell, "On the Dengfeng-Chuntian similarity measure and its application to pattern recognition," Pattern Recognition Letters, vol. 24, no. 16, pp. 3101-3104, 2003.

[15] Z. Liang and P. Shi, "Similarity measures on intuitionistic fuzzy sets," Pattern Recognition Letters, vol. 24, no. 15, pp. 2687-2693, 2003.

[16] Y. Li, D. L. Olson, and Z. Qin, "Similarity measures between intuitionistic fuzzy (vague) sets: a comparative analysis," Pattern Recognition Letters, vol. 28, no. 2, pp. 278-285, 2007.
[17] C. M. Hwang, M. S. Yang, W. L. Hung, and M. G. Lee, "A similarity measure of intuitionistic fuzzy sets based on the Sugeno integral with its application to pattern recognition," Information Sciences, vol. 189, pp. 93-109, 2012.

[18] Z. Xu, "Some similarity measures of intuitionistic fuzzy sets and their applications to multiple attribute decision making," Fuzzy Optimization and Decision Making, vol. 6, no. 2, pp. 109-121, 2007.

[19] Z. S. Xu and J. Chen, "An overview of distance and similarity measures of intuitionistic fuzzy sets," International Journal of Uncertainty, Fuzziness and Knowledge-Based Systems, vol. 16, no. 4, pp. 529-555, 2008.

[20] Z. S. Xu and R. R. Yager, "Intuitionistic and interval-valued intuitionistic fuzzy preference relations and their measures of similarity for the evaluation of agreement within a group," Fuzzy Optimization and Decision Making., vol. 8, no. 2, pp. 123-139, 2009.

[21] W. Zeng and P. Guo, "Normalized distance, similarity measure, inclusion measure and entropy of interval-valued fuzzy sets and their relationship," Information Sciences, vol. 178, no. 5, pp. 1334$1342,2008$.

[22] C. Wei, P. Wang, and Y. Zhang, "Entropy, similarity measure of interval-valued intuitionistic fuzzy sets and their applications," Information Sciences, vol. 181, no. 19, pp. 4273-4286, 2011.

[23] F. E. Boran and D. Akay, "A biparametric similarity measure on intuitionistic fuzzy sets with applications to pattern recognition," Information Sciences, vol. 255, pp. 45-57, 2014.

[24] H. Zhang and L. Yu, "New distance measures between intuitionistic fuzzy sets and interval-valued fuzzy sets," Information Sciences, vol. 245, pp. 181-196, 2013.

[25] J. Li, G. Deng, H. Li, and W. Zeng, "The relationship between similarity measure and entropy of intuitionistic fuzzy sets," Information Sciences, vol. 188, pp. 314-321, 2012.

[26] B. Farhadinia, "A theoretical development on the entropy of interval-valued fuzzy sets based on the intuitionistic distance and its relationship with similarity measure," Knowledge-Based Systems, vol. 39, pp. 79-84, 2013.

[27] D.-F. Li, "Some measures of dissimilarity in intuitionistic fuzzy structures," Journal of Computer and System Sciences, vol. 68, no. 1, pp. 115-122, 2004.

[28] G. A. Papakostas, A. G. Hatzimichailidis, and V. G. Kaburlasos, "Distance and similarity measures between intuitionistic fuzzy sets: a comparative analysis from a pattern recognition point of view," Pattern Recognition Letters, vol. 34, no. 14, pp. 1609-1622, 2013.

[29] S. M. Chen, "Measures of similarity between vague sets," Fuzzy Sets and Systems, vol. 74, no. 2, pp. 217-223, 1995.

[30] D. H. Hong and C. Kim, "A note on similarity measures between vague sets and between elements," Information Sciences, vol. 115, no. 1-4, pp. 83-96, 1999.

[31] F. $\mathrm{Li}$ and $\mathrm{Z}$. Xu, "Similarity measures between vague sets," Journal of Software, vol. 12, no. 6, pp. 922-927, 2001.

[32] Y. Li, Z. Chi, and D. Yan, "Similarity measures between vague sets and vague entropy," Journal of Computer Science, vol. 29, pp. 129-132, 2002.

[33] I. K. Vlachos and G. D. Sergiadis, "Intuitionistic fuzzy information-applications to pattern recognition," Pattern Recognition Letters, vol. 28, no. 2, pp. 197-206, 2007.

[34] C. M. Own, "Switching between type-2 fuzzy sets and intuitionistic fuzzy sets: an application in medical diagnosis," Applied Intelligence, vol. 31, no. 3, pp. 283-291, 2009. 
[35] E. Szmidt and J. Kacprzyk, "A similarity measure for intuitionistic fuzzy sets and its application in supporting medical diagnostic reasoning," in Proceedings of the 7th International Conference on Artificial Intelligence and Soft Computing (ICAISC '04), pp. 388-393, June 2004.

[36] E. Szmidt and J. Kacprzyk, "Intuitionistic fuzzy sets in intelligent data analysis for medical diagnosis," in Computational ScienceICCS 2001, vol. 2074 of Lecture Notes in Computer Science, pp. 263-271, 2001. 


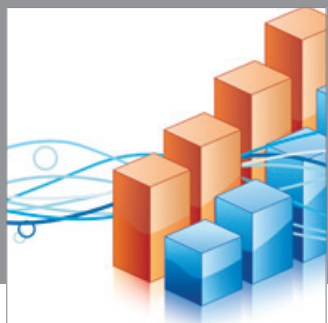

Advances in

Operations Research

mansans

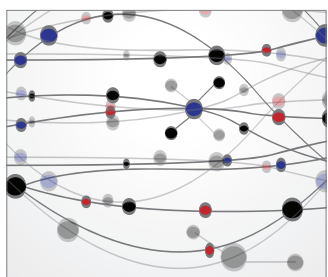

The Scientific World Journal
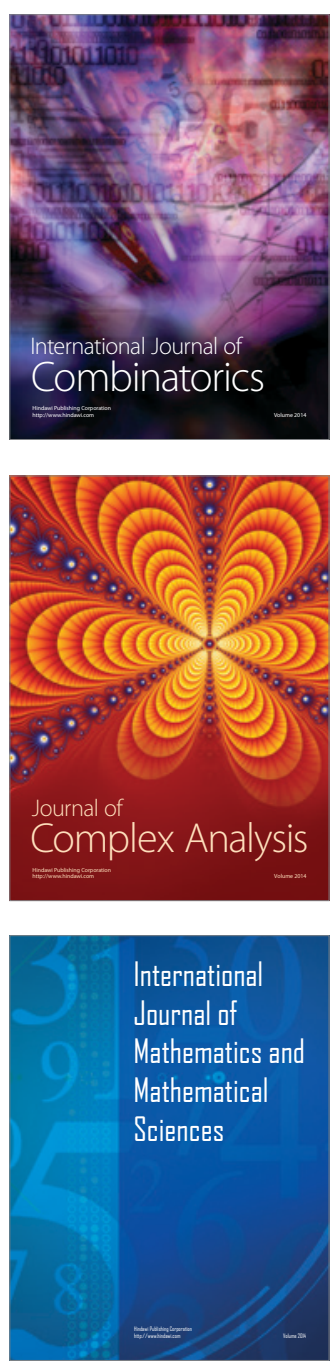
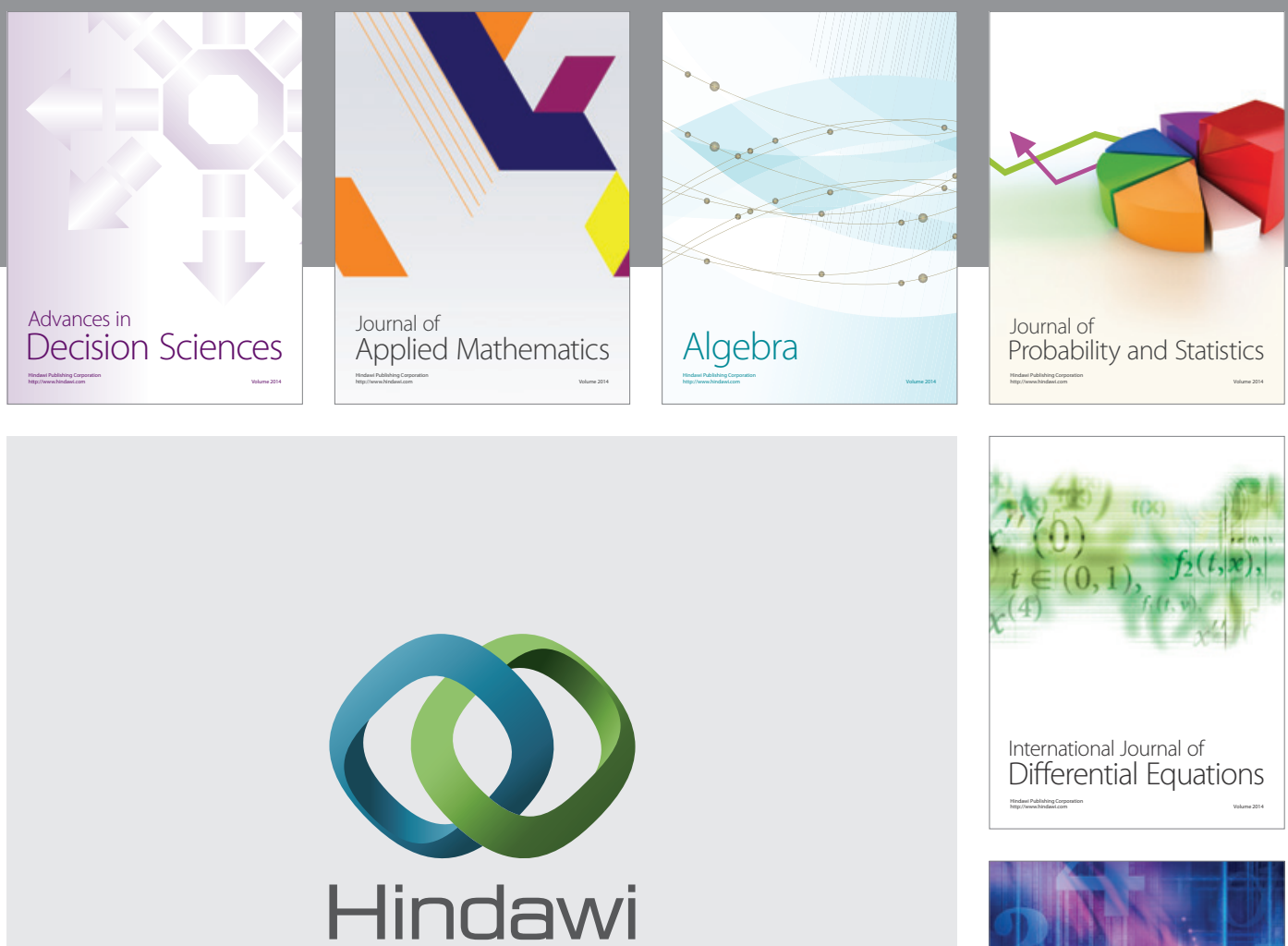

Submit your manuscripts at http://www.hindawi.com
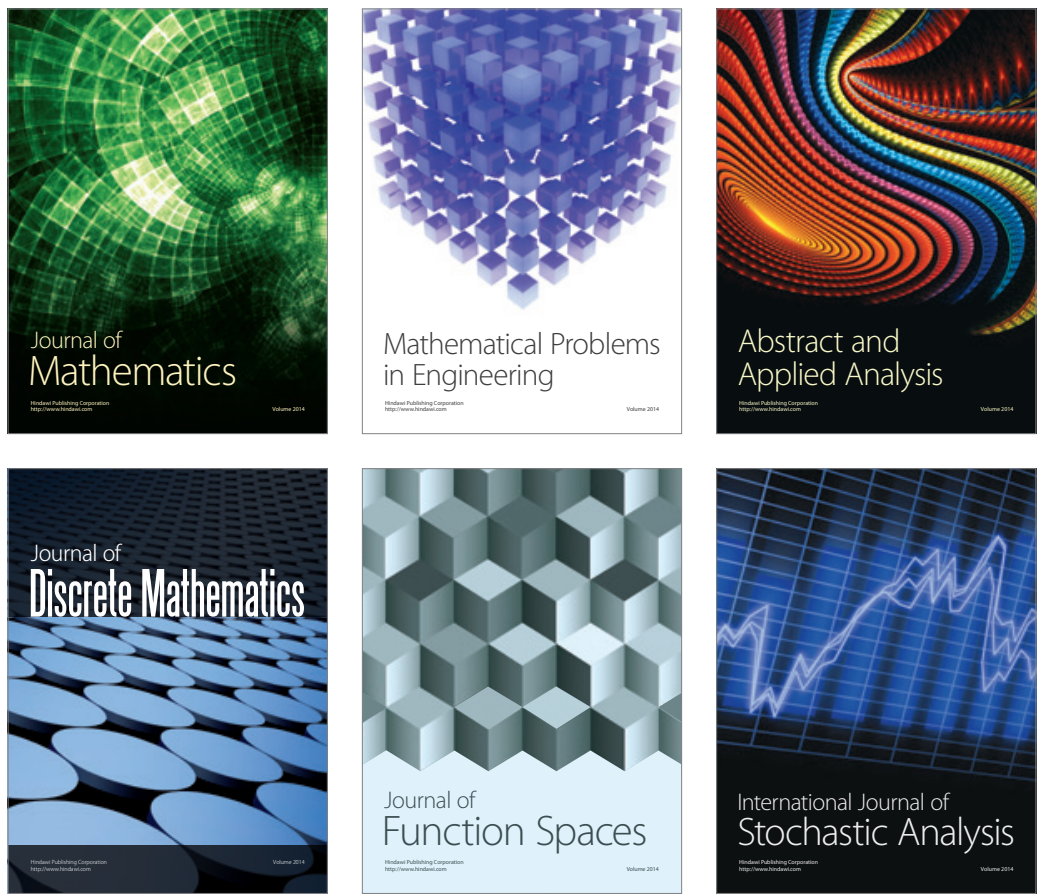

Journal of

Function Spaces

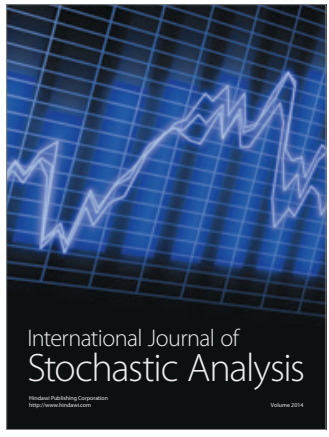

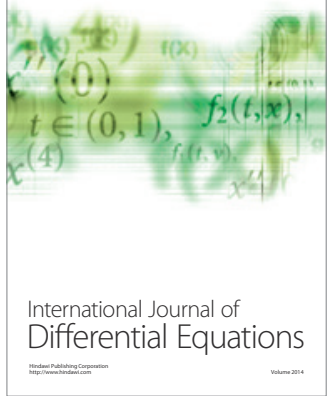
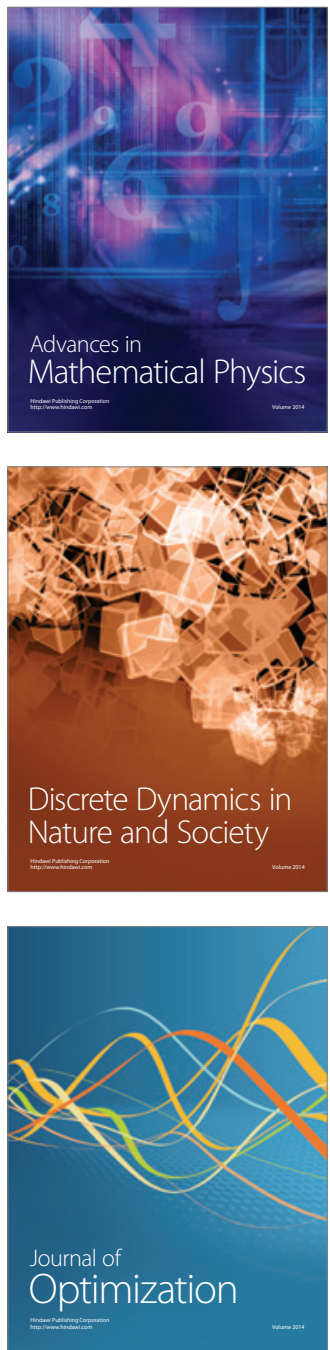